\title{
SHORT ANTENNAS ON A LARGE SPACECRAFT
}

\author{
G. Fischer*, B. Cecconi ${ }^{\dagger}$, J. Bergman ${ }^{\ddagger}$, J. Girard ${ }^{\S}$, G. Quinsac ${ }^{\dagger}$, \\ and J.-E. Wahlund ${ }^{\ddagger}$
}

\begin{abstract}
Short dipole or monopole radio antennas are defined as being small in length relative to the wavelength of the frequency of operation. The reception properties of short linear antennas can be described by the so-called effective length vector which is pointing along the direction of minimum gain in the toroidal radiation pattern. We deal here with such antennas, and additionally the word "short" also means a small antenna with respect to a large spacecraft body. Using numerical computer simulations we calculate the reception properties of an antenna system consisting of three short monopoles positioned on a large spacecraft body in the frequency range of several hundred $\mathrm{kHz}$. It turns out that such a configuration has the major disadvantage that the angular separation between its three effective length vectors is quite small, which would lead to large errors in polarization and direction-finding measurements. We will show ways how to overcome this problem by changing the configuration to an antenna triad consisting of three short dipoles mounted on a boom. The calculations were employed to find a good configuration of the radio antennas for ESA's future JUICE (Jupiter Icy Moons Explorer) mission.
\end{abstract}

\section{Introduction with theory and methods}

In order to retrieve correct data from a radio instrument, it is necessary to know the reception properties of its antenna system. The reception properties of short linear antennas can be described with the so-called effective length vector $\vec{h}_{e f f}$, which relates the measured voltage $V$ at the antenna port to the incident electric field $\vec{E}$ with the simple relation

$$
V=\vec{E} \cdot \vec{h}_{e f f} .
$$

In case of 3 antennas this becomes the matrix equation

$$
-\vec{V}=\mathbf{T} \cdot \vec{E}
$$

\footnotetext{
* Space Research Institute, Austrian Academy of Sciences, Graz, Austria

${ }^{\dagger}$ LESIA, Observatoire de Paris, PSL Research University, CNRS, Meudon, France

$¥$ Swedish Institute of Space Physics, Uppsala, Sweden

$\S$ AIM/IRFU/SAP-CEA Saclay, Université Paris Diderot, France
} 
where $\mathbf{T}$ is the so-called $3 \times 3$ transfer matrix with the effective length vectors of the single antennas as rows [Macher, 2005; Macher et al., 2007], and the vector $\vec{V}$ consists of the voltage drops $V_{i}$ at the 3 ports $i=1,2,3$. With a system of 3 antennas it is principally possible to retrieve the intensity, polarization and incoming direction of the radio wave, but without knowledge of the transfer matrix one would get erroneous values for these physical quantities. This is due to the fact that effective lengths vectors generally do not coincide with the physical antenna directions. The effective antenna direction for a monopole is largely determined by the surface currents on the spacecraft body, against which it is driven. In the quasi-static frequency range, where the wavelength is much larger than the spacecraft's dimension, the effective length vector can be calculated as the integral over all currents divided by the feed current [Collin and Zucker, 1969; Fischer et al., 2001; Macher et al., 2007]. So we need a way to find the current distribution in transmission mode.

4NEC2 (graphical user interface for (4) Numerical Electromagnetics Code, version 2) and ASAP (Antenna Scatterers Analysis Program) are codes that can calculate the current distribution by solving the electric field integral equation (EFIE) with the method of moments [Harrington, 1968]. Both 4NEC2 and ASAP have been released to the public and are freely available on the internet (http://www .qsl.net/4nec2 and http://raylcross . net/asap/). For both programs it is necessary to set up a wire-grid model of the whole spacecraft with the antenna system. Before we will do this in Section 3 with a simple spacecraft model, we will calculate an analytical model with a triad of monopoles above an infinite ground plane in Section 2. This will already lead us to the problem that 3 short monopoles mounted on a large spacecraft body have 3 effective length vectors with an insufficient angular separation between them. All separation angles should be larger than $70^{\circ}$. If this is not the case, the accuracy of radio wave direction-finding is significantly deteriorated [Cecconi and Zarka, 2005; Bergman et al., 2013]. In Section 3 we will also discuss ways how to overcome this problem before we come to the conclusions.

\section{Analytical model}

In the following we model 3 perpendicular monopoles above a perfectly conductive infinite ground plane (see also Bergman et al. [2013]). The horizontal ground plane is the xyplane and the monopoles are mounted symmetrically around the z-axis (see Figure 1). Using spherical coordinates the 3 monopoles have the colatitudes $\theta_{1}=\theta_{2}=\theta_{3}=54.7^{\circ}$ and the azimuth angles $\phi_{1}=0^{\circ}, \phi_{2}=120^{\circ}$, and $\phi_{3}=240^{\circ}$. The current induced by a radio wave in an antenna will have contributions from the direct (primary) wave and the reflected (mirrored) wave. This is equivalent to the primary induced current $(\overrightarrow{J P})$ above the plane and the mirrored current $\left(\overrightarrow{J^{M}}\right)$ below the plane. These currents can be written using the colatitude $\theta$ and the azimuth $\phi$ of the spherical coordinate system:

$$
\begin{gathered}
\vec{J}^{P}=J\left(\sin \theta \cos \phi \overrightarrow{e_{x}}+\sin \theta \sin \phi \overrightarrow{e_{y}}+\cos \theta \overrightarrow{e_{z}}\right) \\
\vec{J}^{M}=J\left(-\sin \theta \cos \phi \overrightarrow{e_{x}}-\sin \theta \sin \phi \overrightarrow{e_{y}}+\cos \theta \overrightarrow{e_{z}}\right)
\end{gathered}
$$

with $\overrightarrow{e_{x}}, \overrightarrow{e_{y}}$, and $\overrightarrow{e_{z}}$ as the unit vectors of the Cartesian coordinate system. Note that the vertical and horizontal components of the primary current are mirrored differently. The 
vertical component $(\mathrm{z})$ does not change its sign due to oppositely charged image charges above and below the xy-plane, which is illustrated by the vertical arrows in Figure 1. However, the horizontal components ( $\mathrm{x}$ and $\mathrm{y}$ ) change their signs, which is the reason for the negative signs of the $\mathrm{x}$ and $\mathrm{y}$ components in $\overrightarrow{J^{M}}$ compared to $\overrightarrow{J^{P}}$ in the equations above. The effective current in one antenna is then the sum of the respective primary current plus the sum of all mirrored currents, the latter given as $\overrightarrow{J^{M}}=\overrightarrow{J_{1}^{M}}+\overrightarrow{J_{2}^{M}}+\overrightarrow{J_{3}^{M}}$. After some algebra (use the relations $\cos \theta_{i}=1 / \sqrt{3}, \sin \theta_{i}=\sqrt{2} / \sqrt{3}, \cos \phi_{1}=1, \sin \phi_{1}=0$, $\cos \phi_{2}=-1 / 2, \sin \phi_{2}=\sqrt{3} / 2, \cos \phi_{3}=-1 / 2, \sin \phi_{3}=-\sqrt{3} / 2$, and $\overrightarrow{J^{M}}=J \sqrt{3} \overrightarrow{e_{z}}$ ) one will find for the effective currents $\overrightarrow{J_{i}^{E}}=\overrightarrow{J_{i}^{P}}+\overrightarrow{J^{M}}$ under the assumption of equally excited antennas $\left(J=J_{1}=J_{2}=J_{3}\right)$ :

$$
\begin{array}{r}
\overrightarrow{J_{1}^{E}}=\overrightarrow{J_{1}^{P}}+\overrightarrow{J^{M}}=J\left(\frac{\sqrt{2}}{\sqrt{3}} \overrightarrow{e_{x}}+\frac{4}{\sqrt{3}} \overrightarrow{e_{z}}\right) \\
\overrightarrow{J_{2}^{E}}=\overrightarrow{J_{2}^{P}}+\overrightarrow{J^{M}}=J\left(-\frac{1}{\sqrt{6}} \overrightarrow{e_{x}}+\frac{1}{\sqrt{2}} \overrightarrow{e_{y}}+\frac{4}{\sqrt{3}} \overrightarrow{e_{z}}\right) \\
\overrightarrow{J_{3}^{E}}=\overrightarrow{J_{3}^{P}}+\overrightarrow{J^{M}}=J\left(-\frac{1}{\sqrt{6}} \overrightarrow{e_{x}}-\frac{1}{\sqrt{2}} \overrightarrow{e_{y}}+\frac{4}{\sqrt{3}} \overrightarrow{e_{z}}\right)
\end{array}
$$

Using the scalar product one can calculate the separation angle $\alpha$ between the various effective current vectors. For any two effective current vectors one will find the result $\cos \alpha=5 / 6$, i.e. $\alpha=33.6^{\circ}$. This is quite far from the separation angle of $90^{\circ}$ between the physical antennas.

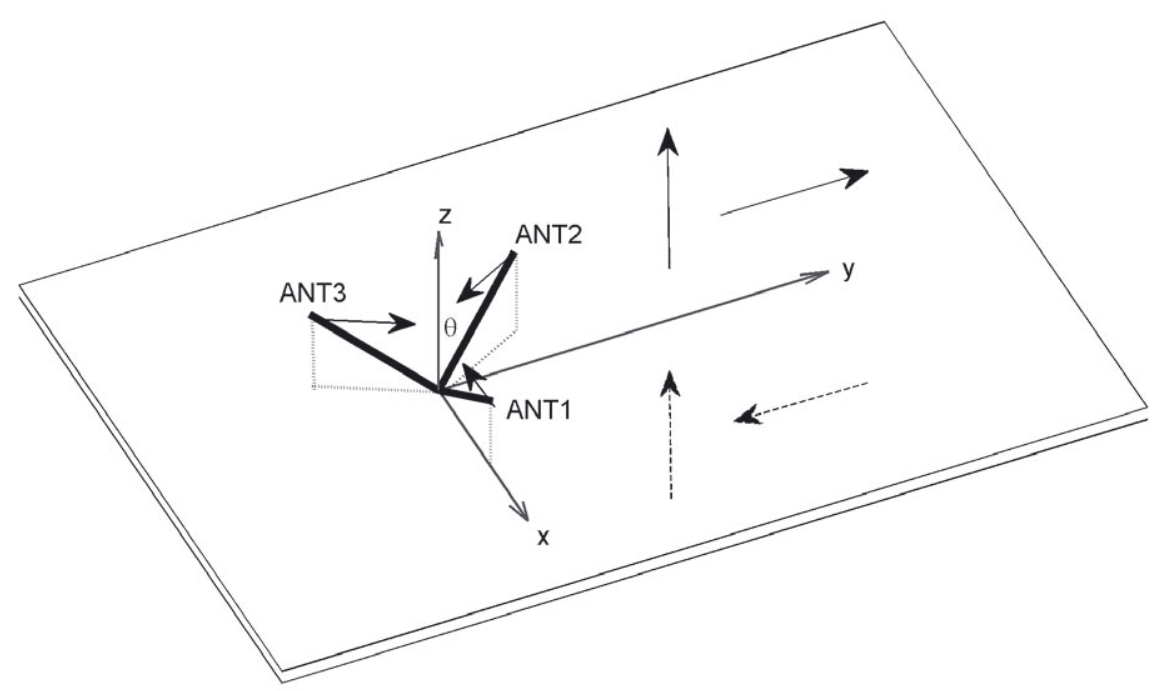

Figure 1: Sketch of the analytical model with 3 monopoles above an infinite ground plane. The arrows along the $y$-axis illustrate how vertical and horizontal currents are mirrored. The arrows at the antennas show the direction towards the z-axis to which the effective antennas are pushed with respect to the physical antennas.

We can also calculate the spherical coordinates of the effective currents (same direction as effective antennas) which yields $\theta_{i, e f f}=\arccos (2 \sqrt{2} / 3)=19.5^{\circ}$, and the effective azimuth 
angles stay the same as the azimuth angles of the real antennas $\left(\phi_{1, \text { eff }}=0^{\circ}, \phi_{2, \text { eff }}=120^{\circ}\right.$, and $\phi_{3, e f f}=240^{\circ}$ ). This means that the effective antennas are moving inwards towards the z-axis with respect to the physical antennas, and their colatitude is reduced from $\theta_{i}=54.7^{\circ}$ to $\theta_{i, e f f}=19.5^{\circ}$. It is interesting to note that the effective antenna of one single monopole (without the influence of the mirrored currents from the 2nd and 3rd antenna) would be aligned with the z-axis. Another interesting point is the question under which circumstances are the effective currents perpendicular to each other? This is the case for $\cos \theta_{i}=1 / \sqrt{33}$ which after some calculation yields $\alpha=90^{\circ}$. However, this means a colatitude of $\theta_{i}=80^{\circ}$, i.e. the antennas are only elevated by $10^{\circ}$ with respect to the ground plane. Such antennas would have a low sensitivity due to the large coupling to the ground. The case of an infinite ground plane is an extreme case, but a large and conductive spacecraft body should have a similar effect on small monopoles mounted on it. The currents on the large surface of the body will tend to push the 3 effective antennas towards each other. This will be shown by numerical modeling in the next section.

\section{$3 \quad$ Numerical models}

Numerical computer models have already been used for the calibration of radio antenna systems of various spacecraft, e.g. for Cassini [Fischer et al., 2001], for STEREO [Macher et al., 2007; Oswald et al., 2009], for Resonance [Sampl et al., 2015], or most recently for Juno [Sampl et al., 2016] and Solar Orbiter (not yet published). One of their main advantages are that they are very flexible, and one can easily change spacecraft configurations, lengths and radii of antennas, values for base capacitances, or calculate the models also for higher frequencies where the effective lengths vectors are not constant anymore.

\subsection{NEC2 model}

The following model consists of 3 monopole antennas of $2.5 \mathrm{~m}$ in length on a spacecraft body, which is a cube with a side length of $2.5 \mathrm{~m}$. The model has 2 large T-shaped solar panels that consist of 4 rectangles with the dimension $4 \mathrm{~m} \times 2 \mathrm{~m}$ each. In Figure 2 parts of the model can be seen. There is also a $5 \mathrm{~m}$ long magnetometer boom at one corner of the cube whose diameter is the same as the wire diameter of the whole model $(5 \mathrm{~mm})$. The computations were done with $4 \mathrm{NEC} 2$ at a frequency of $3.1 \mathrm{MHz}$, and the antenna triad has the following 3 parameters:

- The angle $\beta$ is the opening angle of the antenna triad. It varies from $50^{\circ}$ to $70^{\circ}$ in steps of $5^{\circ}$. Note that orthogonal antennas are present for $\beta=54.7^{\circ} \approx 55^{\circ}$.

- The angle $\alpha$ is the rotation angle along the main axis, and it varies from $0^{\circ}$ to $120^{\circ}$.

- There are 7 steps in distance towards the location of the magnetometer boom (see Figure 2).

Figure 3 shows the results of the calculation for 5 different opening angles $\beta$ (in 5 columns), 9 different rotation angles $\alpha$ (horizontal axes of Figure 3) and 7 different locations of the 


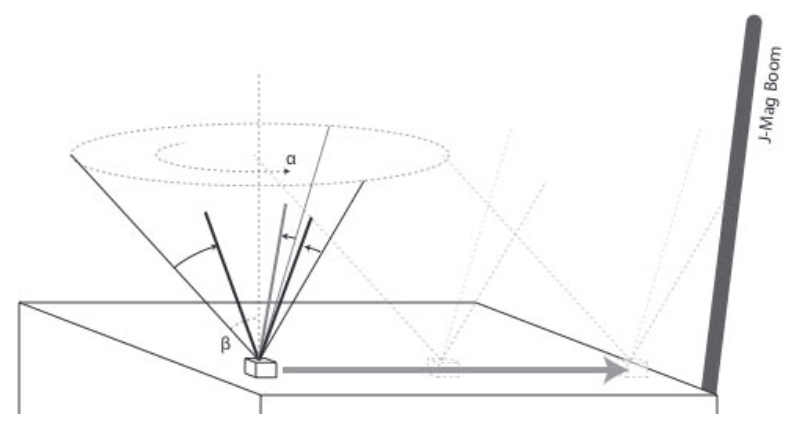

Figure 2: Sketch of 4NEC2 numerical model with the 3 parameters $\alpha$ (rotation angle), $\beta$ (opening angle) and the distance to the boom.
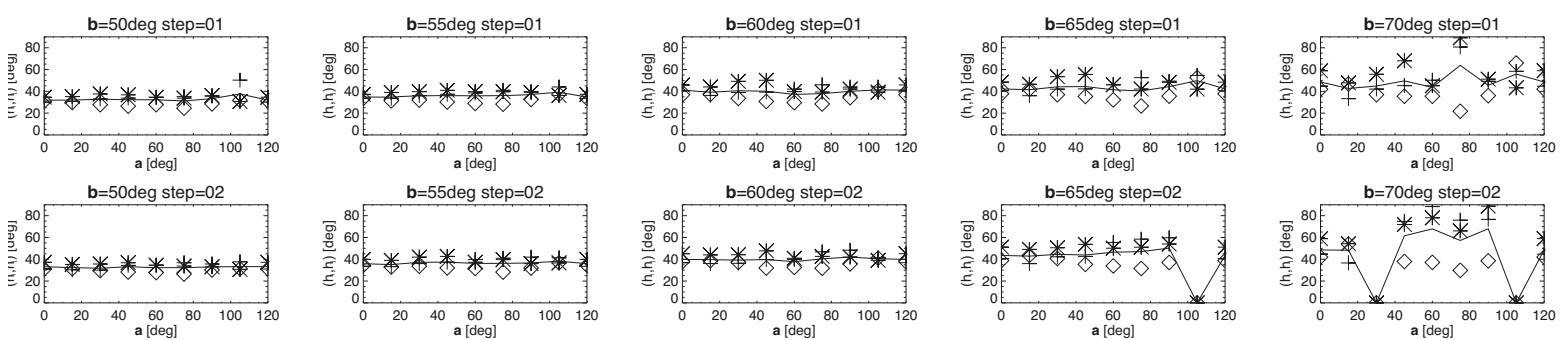

b $=50$ deg step $=03$
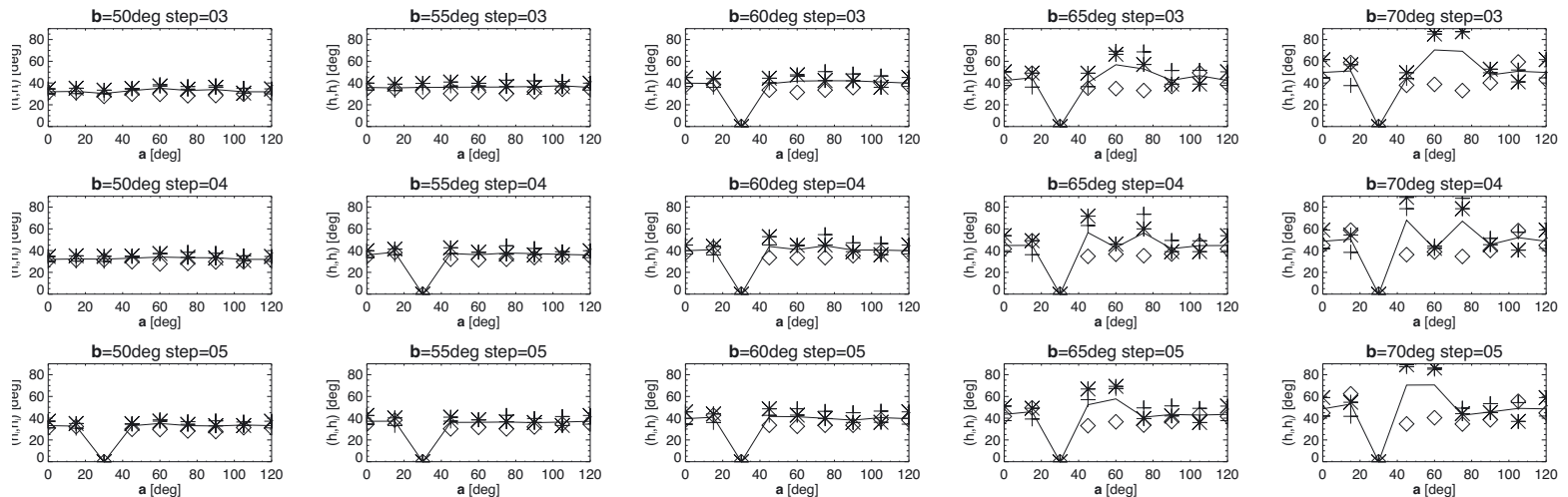

b=55deg step $=06$
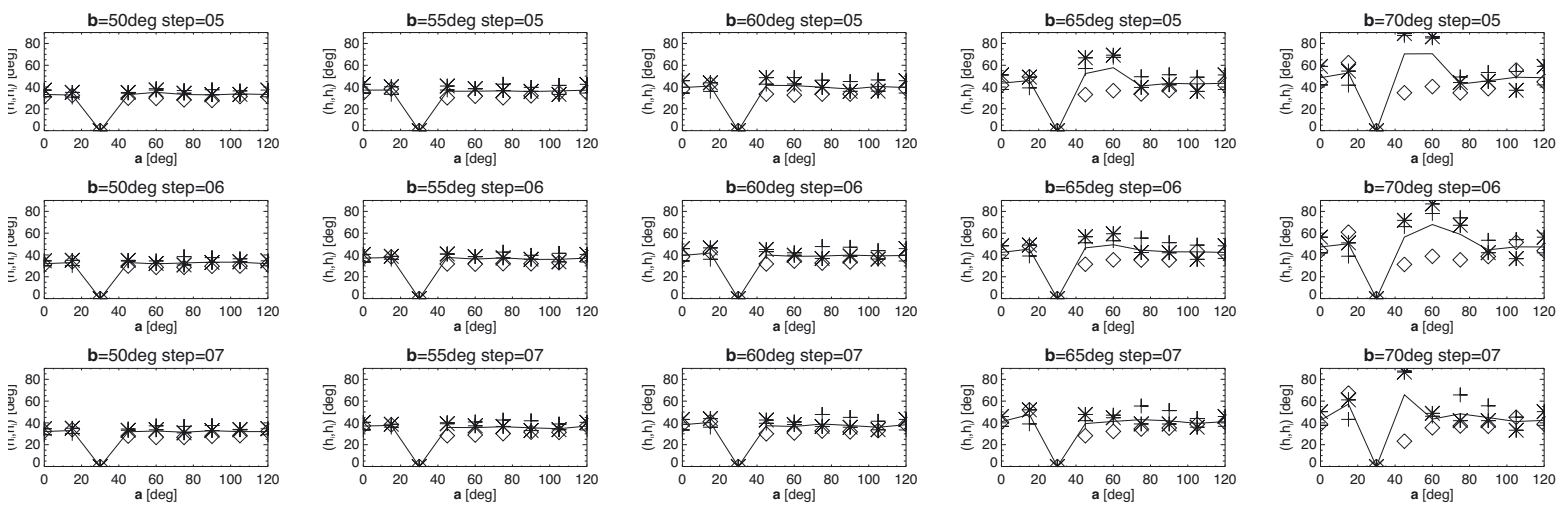

Figure 3: Results of 4NEC2 numerical model. The 3 mutual separation angles between the 3 effective antennas are displayed with 3 different symbols and with the line denoting the average. The simulation results are displayed for 5 different opening angles $\beta$ (in 5 columns from $50^{\circ}$ to $70^{\circ}$ in $5^{\circ}$ steps), 9 different rotation angles $\alpha$ (at horizontal axes from $0^{\circ}$ to $120^{\circ}$ ), and 7 different locations approaching the boom (in 7 rows). In case of an incompatible geometry, e.g. antennas crossing the boom, the data value is zero.

antenna triad with respect to the boom (in 7 rows). This distance to the boom has almost no influence, except for the exceptional position of $\alpha \approx 30^{\circ}$ where one antenna 
is too close to the boom and the computation failed. There is also little variation with rotation angle $\alpha$, except for large opening angles $\beta$. Figure 3 also shows that most angular separation values between the effective antennas are around $40^{\circ}$, which is only slightly more than the separation angle of $33.6^{\circ}$ of our analytical model with 3 monopoles above an infinite ground plane. Also similar to our analytical model of the previous section, only large opening angles $\beta \geq 65^{\circ}$ can lead to larger separation angles between the effective antennas. There are a few cases (last column in Figure 3) where two separation angles are larger than the required $70^{\circ}$, but the third separation angle (diamond symbol in Figure 3 ) is still just around $40^{\circ}$. So there is no antenna configuration here which fulfills our requirements.

\section{$3.2 \quad$ ASAP models}

In this section we will perform further model calculations to overcome the problem of insufficient angular separation between the effective antennas. Our models here consist of a cube as central spacecraft body with a side length of $2.4 \mathrm{~m}$. There are also two large solar panels with a size of $14 \mathrm{~m} \times 2.24 \mathrm{~m}$ each, whose distance to the central body is $0.8 \mathrm{~m}$. The antenna triad again consists of 3 monopoles of $2.5 \mathrm{~m}$ in length with feed points usually close to the common origin. All calculations were performed with ASAP for a frequency of $500 \mathrm{kHz}$ (quasi-static case) and a base capacitance of $C_{b}=20 \mathrm{pF}$.

Figure 4 displays 6 different models with the resulting effective length vectors drawn in color (red for antenna A1, green for A2, and blue for A3). The separation angles between the effective antennas $\left(\alpha_{1}=\angle\left(A 1_{\text {eff }}, A 2_{\text {eff }}\right), \alpha_{2}=\angle\left(A 1_{\text {eff }}, A 3_{\text {eff }}\right), \alpha_{3}=\right.$ $\left.\angle\left(A 2_{\text {eff }}, A 3_{\text {eff }}\right)\right)$ are given for each model. In Figure $4($ a) one can see an orthogonal antenna triad mounted at one corner of the spacecraft body leading to relatively small separation angles. The antenna triad in Figure 4(b) is mounted on one edge of the cube, and the plane of the physical antennas A1 and A2 is tilted by $30^{\circ}$ with respect to the xy-plane (plane of solar panels). The angle between the physical antennas A1 and A2 is $120^{\circ}$, but the angle between the effective antennas is only $\alpha_{1}=37.5^{\circ}$. The configuration in Figure 4(b) can be compared to the similar configuration in Figure 4(c) which has a boom with a length of $5 \mathrm{~m}$ between A1 and A2. Spacecraft parts placed between antennas usually tend to pushes the effective antenna axes further apart [Fischer et al., 2001], and indeed the angular separation increases up to $\alpha_{1}=49.7^{\circ}$. This can be further improved, when increasingly large spacecraft parts are placed between the antennas, like the solar panel in Figure 4(d), which is placed between A1 and A2 when the antenna triad is mounted on an edge below the solar panel. In this case the angular separation increases up to $\alpha_{1}=88.7^{\circ}$, quite close to the optimal value of $90^{\circ}$. However, the two other angles $\alpha_{2}=\alpha_{3}=52.3^{\circ}$ are still smaller than the required minimum separation angle of $70^{\circ}$. We tested about a dozen different configurations with 3 adjacent feed points other than those shown in Figure 4(a) to 4(d). In none of these other cases of adjacent monopoles on the spacecraft body was it possible to retrieve a configuration with all 3 separation angles larger than $70^{\circ}$.

One possible way to increase all three separation angles above $70^{\circ}$ is a separation of the antenna feeds as it is shown in Figure 4(e). The second most simple way would be to 


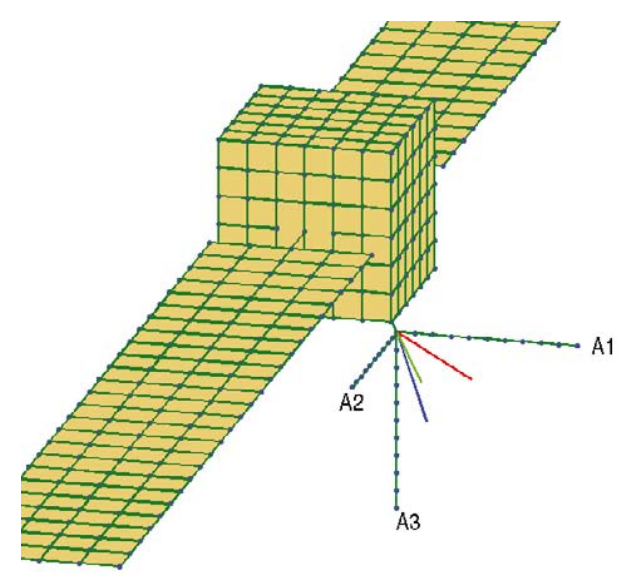

(a)

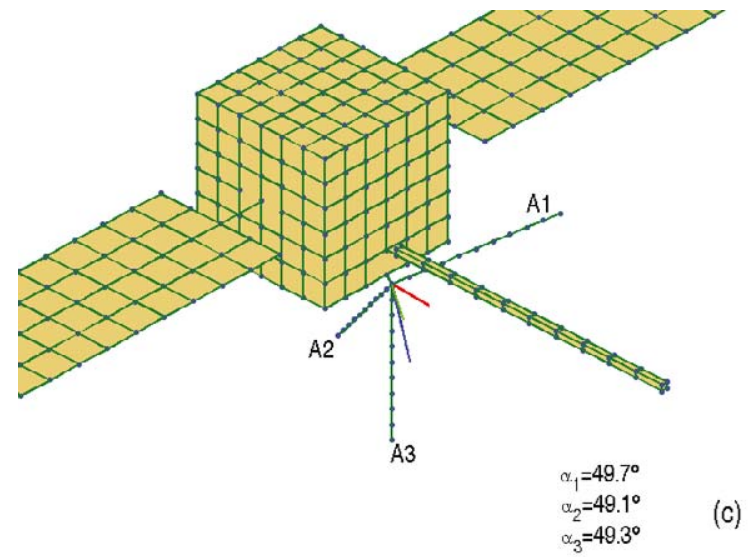

(c)
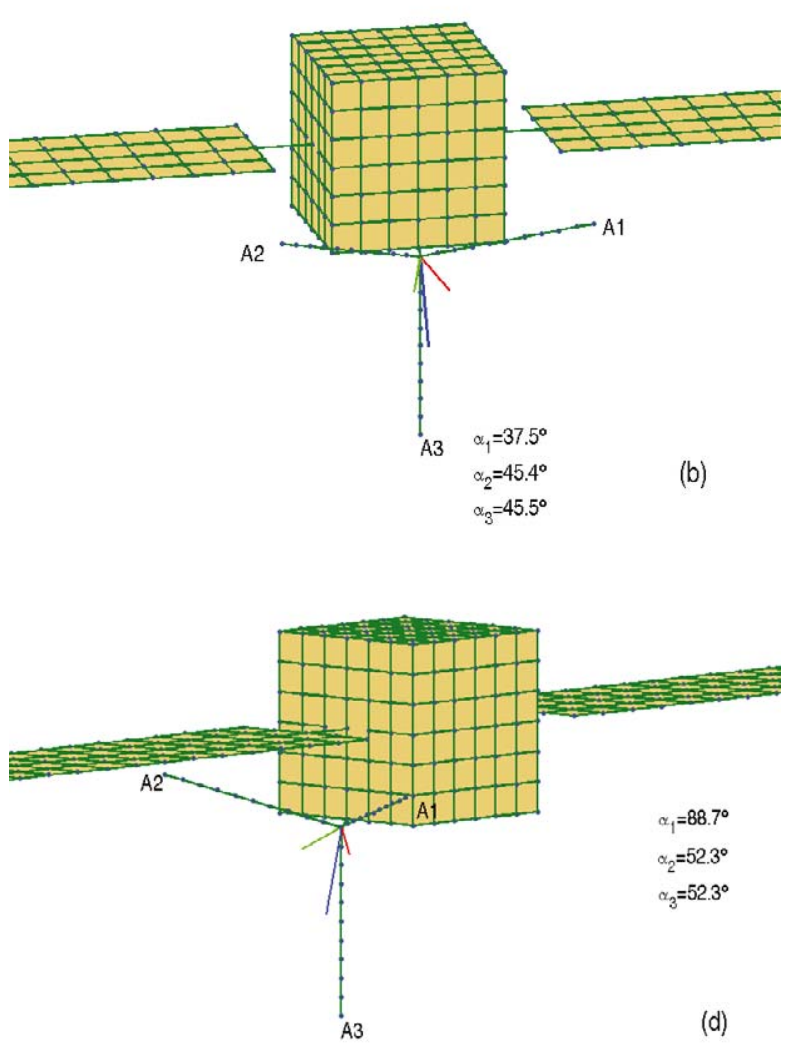

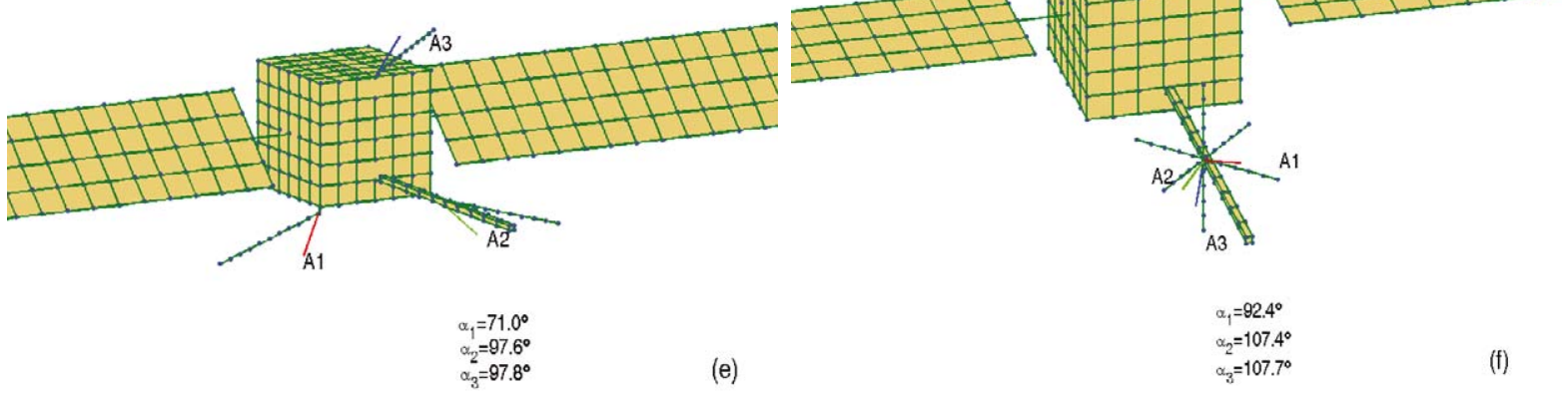

Figure 4: Simulation results for 6 different ASAP models. The effective length vectors are drawn in color (red for antenna A1, green for A2, and blue for A3), and the angles $\alpha_{i}$ denote the angular separations between the effective length vectors.

increase the physical lengths of the antennas (e.g. Cassini RPWS antennas are $10 \mathrm{~m}$ in length), but this option would increase the mass and is not discussed here. In the configuration with separated monopoles in Figure 4(e) the angle between the physical antennas A1 and A2 was set to $144^{\circ}$, and A3 has an angle of $95^{\circ}$ with respect to the plane formed by A1 and A2. The effective antennas of A1 and A2 are still pushed towards each other, but the angle between them is $\alpha_{1}=71.0^{\circ}$, just above our limit. 
However, there are some disadvantages related to this configuration. First, each antenna will need its own pre-amplifier box since long cables between antennas and pre-amplifiers increase the base capacitance and thus decrease the sensitivity. This will increase the mass since 2 additional pre-amplifier boxes are needed. Second, the spatial separation of antennas will introduce phase shifts between the antennas. Depending on the arrival direction and wavelength of the radio wave, these phase shifts will introduce systematic errors in polarization and direction-finding measurements. The final solution shown in Figure 4(f) shows 3 dipole antennas of $2.5 \mathrm{~m}$ in length mounted on a large boom. This configuration also fulfills the angular separation criterion of $\alpha_{i}>70^{\circ}$ for all effective antennas. Here the pre-amplifier can be located in just one box close to the antennas. The disadvantages of this configuration are that additional cables are needed between the pre-amplifier and the box of receivers on the spacecraft, and that the pre-amplifier will need its own thermal control system. Nevertheless, these disadvantages were considered as less serious compared to those from separated monopole antennas on the spacecraft. Therefore, the configuration of dipole antennas mounted on a large boom was chosen for the radio antennas of the RPWI (Radio and Plasma Wave Investigation) instrument [Bergman et al., 2013] on-board ESA's future Jupiter mission JUICE (Jupiter Icy Moons Explorer).

\section{Conclusions}

We used different programs (4NEC2 and ASAP) to model the reception properties of 3 monopole antennas (2.5 $\mathrm{m}$ in length) mounted on a large cubic spacecraft body. It was found that the angular separations between the three effective axes are typically around $40^{\circ}$. This is in line with an angular separation of $33.6^{\circ}$ yielded by an analytical calculation of an orthogonal triad of monopoles placed above a conductive infinite ground plane. The consequences of such small angular separations would be large errors for goniopolarimetric measurements. Ways to overcome this problem would be to place large spacecraft parts between the antennas, to spatially separate the feed points of the monopoles, or to place the antenna triad on a boom. The latter option was the preferred solution for the radio antennas on-board the JUICE mission.

Acknowledgments. This work was supported by the Austrian Research Promotion Agency FFG through project 844347 "Antenna optimization studies for JUICE-RPWI". The French co-authors (B. C., J. G., G. Q.) acknowledge support from Observatoire de Paris, CNRS and CNES. The responsible Editor thanks Alexander Konovalenko and one anonymous reviewer for their help in evaluating this paper.

\section{References}

Bergman, J., et al., Proposal for RWI baseline change, Technical Note 101 to ESA for RPWI/JUICE, 2013. 
Cecconi, B., and P. Zarka, Direction finding and antenna calibration through analytical inversion of radio measurements performed using a system of two or three electric dipole antennas on a three-axis stabilized spacecraft, Radio Sci., 40, RS3003, 2005.

Collin, R.E., and F. J. Zucker, Antenna theory, Part 1, McGraw-Hill, New York, USA, 1969.

Fischer, G., W. Macher, H. O. Rucker, H. P. Ladreiter, D. F. Vogl, and the Cassini/RPWS Team, Wire-grid modeling of Cassini spacecraft for the determination of effective antenna length vectors of the RPWS antennas, in Planetary Radio Emissions $V$, edited by H. O. Rucker, M. L. Kaiser, and Y. Leblanc, Austrian Academy of Sciences Press, Vienna, 347-356, 2001.

Harrington, R. F., Field Computation by Moment Methods, Robert E. Krieger Publishing Company, Malabar, Florida, USA, 1968.

Macher, W., Transfer matrix description of multi-port antennas and its application to the Mars Express/MARSIS radar, PhD Thesis, Graz University of Technology, Austria, 2005 .

Macher, W., T. Oswald, G. Fischer, and H. O. Rucker, Rheometry of multiport spaceborne antennas including mutual antenna capacitances and application to STEREO/WAVES, Meas. Sci. Techn., 18, 3731-3742, 2007.

Oswald, T., W. Macher, H. O. Rucker, G. Fischer, U. Taubenschuss, J.-L. Bougeret, A. Lecacheux, M. L. Kaiser, and K. Goetz, Various methods of calibration of the STEREO/WAVES antennas, Adv. Space Res., 43, 355-364, 2009.

Sampl, M., W. Macher, C. Gruber, T. Oswald, M. Kapper, H. O. Rucker, and M. Mogilevsky, High-frequency performance of electric field sensors aboard the Resonance satellite, Geosci. Instrum. Method. Data Syst., 4, 81-88, 2015.

Sampl, M., W. Macher, T. Oswald, D. Plettemeier, H. O. Rucker, and W. S. Kurth, Juno model rheometry and simulation, Radio Sci., 51, 1627-1635, 2016. 
